\title{
Connecting Cerebral White Matter Lesions and Hypertensive Target Organ Damage
}

\author{
Cristina Sierra, ${ }^{1,2}$ Alfons López-Soto, ${ }^{2}$ and Antonio Coca ${ }^{1}$ \\ ${ }^{1}$ Hypertension Unit, Department of Internal Medicine, Institute of Medicine and Dermatology, Hospital Clinic of Barcelona, IDIBAPS, \\ University of Barcelona, Villarroel 170, 08036 Barcelona, Spain \\ ${ }^{2}$ Geriatric Unit, Department of Internal Medicine, Institute of Medicine and Dermatology, Hospital Clinic of Barcelona, IDIBAPS, \\ University of Barcelona, Villarroel 170, 08036 Barcelona, Spain
}

Correspondence should be addressed to Cristina Sierra, csierra@clinic.ub.es

Received 4 March 2011; Accepted 2 June 2011

Academic Editor: Ana Verdelho

Copyright ( 2011 Cristina Sierra et al. This is an open access article distributed under the Creative Commons Attribution License, which permits unrestricted use, distribution, and reproduction in any medium, provided the original work is properly cited.

Chronic hypertension leads to concomitant remodeling of the cardiac and vascular systems and various organs, especially the brain, kidney, and retina. The brain is an early target of organ damage due to high blood pressure, which is the major modifiable risk factor for stroke and small vessel disease. Stroke is the second leading cause of death and the number one cause of disability worldwide and over $80 \%$ of strokes occur in the elderly. Preclinical hypertensive lesions in most target organs are clearly identified: left ventricular hypertrophy for the heart, microalbuminuria for the kidney, fundus abnormalities for the eye, and intima-media thickness and pulse wave velocity for the vessels. However, early hypertensive brain damage is not fully studied due to difficulties in access and the expense of techniques. After age, hypertension is the most-important risk factor for cerebral white matter lesions, which are an important prognostic factor for stroke, cognitive impairment, dementia, and death. Studies have shown an association between white matter lesions and a number of extracranial systems affected by high BP and also suggest that correct antihypertensive treatment could slow white matter lesions progression. There is strong evidence that cerebral white matter lesions in hypertensive patients should be considered a silent early marker of brain damage.

\section{Introduction}

Chronic hypertension leads to concomitant remodeling of the cardiac and vascular systems, and various organs, especially the brain, kidney, and retina $[1,2]$. Early detection of hypertensive target organ damage is important for moresuccessful prevention of cardiovascular diseases and to improve outcomes $[1,2]$. The brain is an early target for organ damage due to high blood pressure (BP) $[1,2]$, which is the major modifiable risk factor in men and women for ischemic and hemorrhagic stroke [3], as well as small vessel disease $[1,2,4]$ predisposing to lacunar infarction, white matter lesions (WML), and cerebral microbleeds, which are frequently silent $[1,2,5]$.

Stroke is the second leading cause of death and the leading cause of disability worldwide [6]. For each decade of life after the age of 55 years, the stroke rate doubles in both men and women, and $>80 \%$ of strokes occur in people aged $\geq 65$ years [6]. Because of the aging population, the burden of stroke will increase greatly in forthcoming years. The increased vulnerability of elderly people to stroke is associated with changes in the aging brain and also with a higher prevalence of well-documented risk factors for stroke such as hypertension, atrial fibrillation, carotid stenosis, and cardiovascular disease [3].

The clinical significance and pathological substrate of WML are incompletely understood. It is known that WMLs are an important prognostic factor for stroke, cognitive impairment, dementia and death [7]. Cerebral WML are more common and extensive in patients with cardiovascular risk factors, such as hypertension and diabetes mellitus, heart disease, and symptomatic cerebrovascular disease [710]. However, it is controversial whether BP still is associated with WML in patients manifesting vascular disease. Vlek et al. [11] showed in 1030 patients manifesting vascular disease (including cerebrovascular disease, coronary 
heart disease, peripheral arterial disease, abdominal aortic aneurysm), that BP was not associated with the presence of WML, irrespective of the presence of diabetes mellitus or the localisation of vascular disease.

Older age and hypertension are constantly reported to be the main risk factors for cerebral WML $[4,10]$. Hypertensive patients have a higher rate and extension of cerebral WML compared with normotensives $[10,12]$, but treated, controlled hypertensive patients have a lower prevalence of WML than both untreated and treated but uncontrolled hypertensives [12]. A magnetic resonance imaging (MRI) substudy of the randomized PROGRESS trial of BP lowering with perindopril versus placebo in normotensive and hypertensive subjects with cerebrovascular disease found that the mean total volume of new WML was significantly reduced in the active treatment group compared with placebo [13]. A post hoc analysis found that greatest beneficial effect of antihypertensive therapy on WML progression was observed in patients with severe WML at study entry. Godin et al. [14] have recently shown that, in a prospective population-based cohort of 1319 subjects aged $\geq 65$ years, BP at baseline and changes in BP over a 4-year follow-up are strong predictors of WML volume progression independently of potential confounders and suggest that correct antihypertensive treatment could slow WML progression. Interestingly, the Cardiovascular Health Study [15] has been recently shown that diastolic BP had no effect on ischemic stroke incidence in elderly patients with low WML levels but had a marginally significant J-curve relationship with ischemic stroke in elderly with high WML levels. Indeed, in elderly individuals with lowgrade WML, low DBP may not pose a risk for ischemic stroke. However, in high-grade WML, ischemic stroke risk may increase in diastolic BP less than $69 \mathrm{mmHg}$ but is highest more than $80 \mathrm{mmHg}$. The main current hypothesis concerning the association between high BP and WML is that long-standing hypertension causes lipohyalinosis of the media and thickening of the vessel walls, with narrowing of the lumen of the small perforating arteries and arterioles that nourish the deep white matter. The perforating vessels, which originate in the cortical and leptomeningeal arteries, have a relatively poor anastomotic system, which makes the white matter particularly vulnerable to cerebral ischemia. Low BP has also been reported to be a risk factor for WML [4]. Vuorinen et al. [16] showed in a 20-year follow-up study that risk of late-life WML was related to midlife hypertension, and hypertension from midlife to late life also increased the risk of WML. In addition, an association with WML was seen for decreasing BP (hypertension at midlife but not at late life) even after controlling for antihypertensive treatment. Authors speculate that the decline in BP could be secondary to dementia-related processes, when structures involved in BP regulation become affected. It has been shown in elderly people that severe WMLs frequently coexist with medial temporal lobe and global brain atrophy [17].

There is strong evidence that cerebral WML in hypertensive patients should be considered a silent early marker of brain damage [2]. Early atherosclerotic changes in the cerebrovascular system, ultimately leading to incident stroke and cognitive impairment or dementia $[4,7]$, could be mirrored by hypertensive target organ damage in the cardiac, renal, retinal, and vascular and other systems. Therefore, assessment of hypertensive target organ damage in these systems may be indicative of the extent of vascular disease in less easily accessible sites such as the brain.

\section{Cerebral WML, Left Ventricular Hypertrophy, and Geometry}

Several forms of heart damage, such as heart failure, coronary heart disease, and cardiac arrhythmias have been associated with WML [10] although the underlying pathogenetic mechanisms are not clear. Studies have reported that echocardiographically determined left ventricular hypertrophy (LVH) is an independent risk factor for cardiovascular morbidity and mortality in essential hypertensive patients [18, 19]. Bikkina et al. [20] demonstrated that left ventricular mass (LVM) was associated with an increased risk of cerebrovascular events such as stroke and transient ischemic attack in an elderly cohort from the Framingham Heart Study. It is suggested that left ventricular geometric patterns add prognostic information on the development of cardiovascular disease [21] and the presence of extracardiac target organ damage in essential hypertension [22, 23]. Hypertensive patients with concentric LVH have more-advanced target organ damage, including renal $[22,23]$ and retinal $[23]$ involvement, than patients with other patterns of left ventricular geometry. Some studies have found an association between LVH and cerebral WML [23-28], but others have not [29]. We found a close relationship between silent WML and concentric LVH in middle-aged untreated essential hypertensive patients, with WML being more common among patients with concentric LVH [27]. This association was independent of the degree of BP elevation. The mechanisms connecting LVH and cerebrovascular damage are unclear and might reflect long-term exposure to genetic, hormonal, or metabolic factors in addition to $\mathrm{BP}$ [30]. It is difficult to differentiate the relative role of high $\mathrm{BP}$ from the direct contribution of $\mathrm{LVH}$ to the increased risk of cerebrovascular disorders, and longitudinal studies are necessary. However, detection of cardiac hypertrophy may help to identify patients at risk of cerebrovascular injury [2].

\section{Cerebral WML and Early Renal Alteration}

The brain and the kidney are highly vascular structures that respond to diseases such as hypertension and diabetes mellitus in similar ways at the microscopic level. In nephrosclerosis, gradual alterations in the renal endothelial cells, glomeruli, and interstitial spaces lead to glomerular leakage of serum proteins into the urine. If a similar process were occurring at the endothelial level in cerebral microvessels, serum proteins would pass into the cerebral extracellular space. Neuropathological studies show that white matter hyperintensities may represent enlarged perivascular spaces and perivascular demyelination, among other mechanisms [31]. These changes are what might be expected if the cerebral extracellular spaces were exposed to proinflammatory 
proteins which, in healthy individuals, should remain within the vascular space. While there is no direct proof that this process occurs, some studies have shown that WMLs are associated with microalbuminuria [32-34].

It has been suggested that the brain and the kidney have a common, unique way of reacting to fluctuations in BP and flow due to similar low-resistance vascular beds. High-pressure fluctuations in the carotid, vertebral, and renal arteries, together with the turbulent flow, expose the small vessels of these two organs to pressure and flow fluctuations that may explain the microvascular damage and the resulting renal failure and neurological and cognitive alterations [35]. In addition, chronic renal disease (CRD) has been linked with proinflammatory and procoagulant states [36], which may contribute to WML $[37,38]$. Therefore, information on renal microvascular damage may provide information on cerebral damage. Small vessel disease is a systemic condition which is induced by aging and exacerbated by vascular risk factors, especially hypertension, and affects the brain and other systems.

In the kidney, the damage markers are albuminuria/proteinuria and the estimated glomerular filtration rate (eGFR), which shows the functional reduction. Khatri et al. [39] studied 615 stroke-free subjects (mean age: 70 years) and found that the prevalence of silent WML was independently associated with CRD (people with eGFR between 15-60 $\mathrm{mL} / \mathrm{min}$ per $1.73 \mathrm{~m}^{2}$ when compared to people with $60-90 \mathrm{~mL} / \mathrm{min}$, and $>90 \mathrm{~mL} / \mathrm{min}$ ) even after adjustment for age, sex, ethnicity, education, and vascular risk factors [24]. The Rotterdam study [40] of 484 subjects (mean age: 73.4 years; mean systolic BP: $145.7 \mathrm{mmHg}$; mean eGFR: $54.8 \mathrm{~mL} / \mathrm{min} / 1.73 \mathrm{~m}^{2}$ ) found that individuals with a lower eGFR had more WML.

Takahashi et al. [41] recently studied 2,103 asymptomatic individuals with a younger mean age of 56 years and found that the prevalence of subcortical WML and periventricular WML correlated significantly with lower eGFR; in subgroups with eGFR $\geq 90,60-89$, and $<60 \mathrm{~mL} / \mathrm{min} / 1.73 \mathrm{~m}^{2}$, respectively, the prevalences were subcortical WML: $18 \%$, $21 \%$, and $37 \%$, respectively; and periventricular WML: $7 \%$, $10 \%$, and $21 \%$, respectively. Mean age differed significantly between groups (mean 51, 55, 63 years, resp.), and BP $\geq$ $130 / 85 \mathrm{mmHg}$ was significantly more prevalent as eGFR rose.

Wada et al. [33], in a study of 625 Japanese individuals aged $>61$ years, found that subjects with lower eGFR had higher grades of silent WML, that mean grades of WML were greater in subjects with albuminuria than in those without, and that age and the prevalence of hypertension were significantly higher in individuals with higher grades of WML. Knopman et al. [32] also found an independent association between microalbuminuria and WML in 1251 asymptomatic individuals (mean age: 63.8 years, $78 \%$ with hypertension).

\section{Cerebral WML and Retinal Microvascular Abnormalities}

The retina offers a unique, noninvasive, and easily accessible window to study the microvascular etiology of cerebrovascular disease. Retinal and cerebral small vessels share similar embryological origins, anatomical features, and physiological properties [42].

Retinal microvascular abnormalities, such as microaneurysms, retinal hemorrhages, soft and hard exudates, arteriovenous nicking, and retinal focal arteriolar narrowing, usually result from small-vessel damage due to aging and high BP [43].

Arteriolar narrowing may be a sign of hypertension, a history of hypertension in the last three to six years, or a risk factor for the onset of hypertension in normotensive individuals [44]. Large prospective studies (the ARIC, Beaver Dam, Blue Mountains Eye, and Rotterdam studies) have found that reduced arteriolar diameter is an independent risk factor for developing hypertension within 3 to 10 years in normotensive individuals [43].

Studies have shown that retinal microvascular flow is reduced in persons with WML and lacunar infarction [45] and that retinal and cerebral arterioles share a similar histopathology in patients dying from stroke [46]. In the ARIC study, retinal abnormalities were associated with concurrent BP [47]. In a cohort of 1684 asymptomatic people aged 5172 years from the ARIC study, individuals with WML were more likely to have retinal microvascular abnormalities [48]. In general, in this study, WMLs were significantly associated with increasing age, black ethnicity, and-after adjusting for age, sex, and ethnicity — with higher BP and increased carotid intima-media thickness (IMT). Retinopathy was significantly associated with black ethnicity and, after similar adjustment, with higher systolic BP, fasting glucose level, diabetes mellitus, and increased carotid IMT [48]. After a follow-up of 10 years, retinal microvascular abnormalities measured at baseline were prospectively associated with a long-term risk of subclinical cerebrovascular disease on MRI, independent of conventional risk factors in this population-based cohort of middle-aged persons without clinical stroke [49]. The authors suggested that retinal microvascular abnormalities are early and, possibly, more sensitive markers of subclinical cerebral small-vessel disease before radiological and clinical manifestations become apparent. In a cohort of 1717 people from the Cardiovascular Health Study [50] (mean age: 78.3 years; hypertension: $56.3 \%$; diabetes mellitus: $13 \%$; previous cardiovascular disease, including stroke: $23.8 \%$ ) associations were found between WML grade, prevalent lacunar infarct, and a lower arteriovenous ratio. In 174 patients without a history of transient ischemic attack or stroke before followup, WMLs worsening on MRI 5 years later and incident lacunar infarct were associated with a lower arteriovenous ratio [50].

In light of data reporting that persons with a smaller arteriolar-to-venular ratio tended to have more WMLs on MRI, the Rotterdam study evaluated whether this could be due to arteriolar narrowing or venular dilatation [51]. In a population-based cohort study of 490 people without dementia (age 60-90 years; mean age: 68.4 years; mean systolic BP: $136.7 \pm 19.9 \mathrm{mmHg}$; diabetic patients: $6.3 \%$ ), cerebral small vessel disease was evaluated at baseline. A mean of 3.3 years later, 279 persons had a second MRI. Lacunar infarcts and WML at baseline, and incident infarct and changes in periventricular and subcortical WML and progression, were 
evaluated in the follow-up. Neither venular nor arteriolar diameters were related to the severity of cerebral small vessel disease. Larger venular diameters were, however, associated with a marked progression of cerebral small vessel disease [51]. It has been hypothesized that retinal venular dilatation occurs in response to retinal hypoxia and venular dilatation has been also described as one of the earliest changes in diabetic retinopathy.

Retinal microcirculation abnormalities, including retinopathy, reduced arteriolar diameter and, increased venular caliber are widely observed in the general population [43]. The abnormalities observed in retinopathy may reflect disorders of the retinal vascular wall, endothelial dysfunction, and inflammation secondary especially to diabetes mellitus, age, hypertension, obesity, and metabolic disorders. The decreased arteriolar diameter signals the presence of hypertension (current or old) and the risk of hypertension onset. An increased venular diameter has been associated with diabetes mellitus, obesity, and metabolic disorders. All these abnormalities have been associated with WML.

\section{Cerebral WML and Blood Vessels}

Ultrasonographic findings of increased atherosclerotic plaques and carotid artery IMT are regarded as the subclinical markers of early atherosclerosis and are associated with nonmodifiable and modifiable risk factors and the subsequent risk of new or recurrent stroke [52]. Some studies have found that the severity of IMT and the presence of plaques in the carotid arteries are also predictive of WML [53-56], although another study in the elderly did not [57]. This association between large and small vessel disease may well be mediated via common intermediary risk factors such as hypertension.

It is known that the arterial system gradually stiffens due to the combined effects of aging, high BP, and other vascular risk factors. Increased arterial stiffness results in characteristic increased impedance and pulse wave velocity (PWV) in the aorta, which increases systolic and pulse pressure (PP) centrally. Stiff arteries cause high PP and pulsatile flow to be transmitted to distal organs during systole, damaging the cerebral microvasculature. It has been hypothesized that cerebral microvascular disease results from the damaging forces of abnormal flow pulsations extending into small cerebral arteries as a consequence of arterial stiffening.

In a homogeneous sample of never-treated hypertensive patients aged 50-60 years, after exclusion for known risk factors for cerebrovascular damage, such as diabetes mellitus or significant alcohol intake, Sierra et al. [58] found an association between higher PP (including office, ambulatory 24 hours, daytime and nighttime estimates) as a measure of arterial stiffness and WML. In the elderly, it has recently been shown that brachial PP is associated with WML [59].

Laurent et al. [60] found that aortic stiffness, assessed by carotid-femoral PWV using applanation tonometry, the gold standard for arterial stiffness measurement, was an independent predictor of fatal stroke in patients with essential hypertension. Whether the risk of stroke is mediated by largeand/or small-vessel disease is not clear, but the previously reported increased risk of stroke when preclinical cerebral microvascular disease (WML, silent lacunar infarcts, and/or cerebral microbleeds) is present suggests small-vessel disease involvement [4, 7]. Henskens et al. [61] studied 167 hypertensive subjects (mean age: 51.8 years) and found that higher PWV was significantly associated with a greater volume of WML and lacunar infarcts, but not with cerebral microbleeds after multivariate analyses adjusted for age, sex, brain volume, mean BP, and heart rate. This suggests that aortic stiffness is independently associated with the manifestations of cerebral small-vessel disease in hypertensive patients and links systemic large- to cerebral small-artery disease. With respect to radial arterial pulse wave analysis, Shrestha et al. [62] studied 179 unselected patients with a mean age of 66 years and found that central systolic BP values, measured by radial applanation tonometry, were more closely associated with WML than brachial systolic BP. In this study, the augmentation index did not correlate with the presence of WML.

\section{Cerebral WML, Inflammation, and Endothelial Function}

The role of inflammation in atherosclerosis and stroke has received increasing attention as basic and clinical research has provided evidence that inflammatory mechanisms play a central role in the pathogenesis and progression of atherosclerosis, plaque rupture, thrombosis, and stroke [38]. Endothelial dysfunction contributes to the initiation of atherosclerotic lesions. Whether inflammatory processes, apart from their involvement in large-vessel disease, are also involved in the development and consequences of cerebral small-vessel disease is still poorly understood.

Some studies have shown a relationship between WML and markers of inflammation, such as C-reactive protein [63], while others have not $[64,65]$. A similar relationship has also been found with plasma homocysteine levels [66], lipoprotein-associated phospholipase A2 (Lp-PLA2), and myeloperoxidase (MPO) [67]. Plasma markers of endothelial dysfunction such as intercellular adhesion molecule-1 (ICAM-1) and P-selectin have also been associated with WML $[68,69]$. It has recently been suggested that endothelial activation associated with small vessel disease is accompanied by enhanced levels of tissue plasminogen activator (tPA) and low levels of plasminogen activator inhibitor type 1 (PAI-1) when lacunar strokes are associated with WML, suggesting that differences in the activity of components of the fibrinolytic system might contribute to the development of WML [70].

Inspite of their small simple size one study has reported that altered vascular function and structure in subcutaneous small arteries of patients with late-life depression are related to cerebral small vessel disease, including WML, and basal ganglia and infratentorial changes [71]. The study was performed in 16 patients with late-life depression $(68.8 \%$ hypertensives) who were compared with 15 controls $(80 \%$ hypertensives). Small arteries were isolated and studied using pressure myography after subcutaneous gluteal fat biopsy. 
In summary, there is some evidence to show that inflammation, impaired endothelial function, and abnormal wall growth are involved in the pathogenesis of WML.

Rizzoni et al. [72] found that the small cerebral arteries of patients with essential hypertension had an increased media/ lumen ratio compared with normotensive individuals, similar finding to those previously observed in subcutaneous small arteries. Structural alterations of cerebral small vessels were assessed in 13 hypertensive patients and 15 normotensive individuals undergoing neurosurgery for benign or malign tumors. A small portion of morphologically normal cerebral tissue was excised from surgical samples and examined, and cerebral small resistance arteries were dissected and mounted on an isometric and isobaric myograph. An increased media-to-lumen ratio of subcutaneous small resistance arteries has also been shown to predict the development of cardio-cerebrovascular events in hypertensive patients [73].

\section{Conclusion}

Evidence on the prognostic role of subclinical organ damage continues to grow. Early detection of hypertensive target organ damage is important in order to prevent cardiovascular diseases more successfully and improve patients' outcomes $[1,2]$.

After age, hypertension is the most-important factor for the development of cerebral WML $[4,10]$, which are an important prognostic factor for stroke, cognitive impairment, dementia, and death [7]. Various studies have shown an association between WML and a number of extracranial systems affected by high BP. There are findings that suggest that correct antihypertensive treatment could efficiently slow WML progression [11-13]. Strong evidence suggests that cerebral WML in hypertensive patients should be considered a silent early marker of brain damage.

The latest Reappraisal of the European Guidelines on hypertension management [2] reports that, in a group of 192 untreated hypertensive patients (aged 18-90 years) without overt cardiovascular disease, silent cerebrovascular lesions (WML, lacunar infarcts, cerebral microbleeds) were even more prevalent $(44 \%)$ than cardiac $(21 \%)$ and renal $(26 \%)$ subclinical damage and frequently occur in the absence of other signs of organ damage [5]. Similarly, $58 \%$ of patients with demonstrable cardiac or renal damage or both had silent cerebrovascular lesions.

With MRI being increasingly used for diagnostic procedures, investigation of silent cerebrovascular disease will become more frequent in prognostic and therapeutic studies in hypertension. In the meantime, target organ damage in other systems may aid the evaluation of early hypertensive brain damage.

\section{References}

[1] G. Mancia, G. De Backer, A. Dominiczak et al., "2007 guidelines for the management of arterial hypertension: the task force for the management of arterial hypertension of the European Society of Hypertension (ESH) and of the European
Society of Cardiology (ESC)," Journal of Hypertension, vol. 25, no. 6, pp. 1105-1187, 2007.

[2] G. Mancia, S. Laurent, E. Agabiti-Rosei et al., "Reappraisal of European guidelines on hypertension management: a European Society of Hypertension Task Force documental," Blood Pressure, vol. 27, pp. 2121-2158, 2009.

[3] L. B. Goldstein, C. D. Bushnell, R. J. Adams et al., "Guidelines for the primary prevention of stroke: a guideline for healthcare professionals from the American Heart Association/American Stroke Association," Stroke, vol. 42, pp. 517-584, 2010.

[4] L. Pantoni, "Cerebral small vessel disease: from pathogenesis and clinical characteristics to therapeutic challenges," The Lancet Neurology, vol. 9, no. 7, pp. 689-701, 2010.

[5] L. H. Henskens, R. J. van Oostenbrugge, A. A. Kroon, P. A. Hofman, J. Lodder, and P. W. De Leeuw, "Detection of silent cerebrovascular disease refines risk stratification of hypertensive patients," Journal of Hypertension, vol. 27, no. 4, pp. 846853, 2009.

[6] A. Di Carlo, "Human and economic burden of stroke," Age and Ageing, vol. 38, no. 1, pp. 4-5, 2009.

[7] S. Debette and H. S. Markus, "The clinical importance of white matter hyperintensities on brain magnetic resonance imaging: systematic review and meta-analysis," British Medical Journal, vol. 341, p. c3666, 2010.

[8] L. J. Launer, "Epidemiology of white matter lesions," Topics in Magnetic Resonance Imaging, vol. 15, no. 6, pp. 365-367, 2004.

[9] B. van Harten, J. M. Oosterman, B. J. Potter Van Loon, P. Scheltens, and H. C. Weinstein, "Brain lesions on MRI in elderly patients with type 2 diabetes mellitus," European Neurology, vol. 57, no. 2, pp. 70-74, 2007.

[10] L. Pantoni and J. H. Garcia, "The significance of cerebral white matter abnormalities 100 years after Binswanger's report: a review," Stroke, vol. 26, no. 7, pp. 1293-1301, 1995.

[11] A. L. Vlek, F. L. Visseren, L. J. Kappelle et al., "Blood pressure and white matter lesions in patients with vascular disease: the SMART-MR study," Current Neurovascular Research, vol. 6, no. 3, pp. 155-162, 2009.

[12] D. Liao, L. Cooper, J. Cai et al., "Presence and severity of cerebral white matter lesions and hypertension, its treatment, and its control. The ARIC study," Stroke, vol. 27, no. 12, pp. 22622270, 1996.

[13] C. Dufouil, J. Chalmers, O. Coskun et al., "Effects of blood pressure lowering on cerebral white matter hyperintensities in patients with stroke. The PROGRESS magnetic resonance imaging substudy," Circulation, vol. 112, no. 11, pp. 16441650, 2005.

[14] O. Godin, C. Tzourio, P. Maillard, B. Mazoyer, and C. Dufouil, "Antihypertensive treatment and change in blood pressure are associated with the progression of white matter lesion volumes: the three-city (3C)-dijon magnetic resonance imaging study," Circulation, vol. 123, pp. 266-273, 2011.

[15] S. Reshef, L. Fried, N. Beauchamp, D. Scharfstein, D. Reshef, and S. Goodman, "Diastolic blood pressure levels and ischemic stroke incidence in older adults with white matter lesions," Journals of Gerontology A, vol. 66, pp. 74-81, 2011.

[16] M. Vuorinen, A. Solomon, S. Rovio et al., "Changes in vascular risk factors from midlife to late life and white matter lesions: a 20-year follow-up study," Dementia and Geriatric Cognitive Disorders, vol. 31, pp. 119-125, 2011.

[17] T. den Heijer, L. J. Launer, N. D. Prins et al., "Association between blood pressure, white matter lesions, and atrophy of the medial temporal lobe," Neurology, vol. 64, no. 2, pp. 263267, 2005. 
[18] P. N. Casale, R. B. Devereux, M. Milner et al., "Value of echocardiographic measurement of left ventricular mass in predicting cardiovascular morbid events in hypertensive men," Annals of Internal Medicine, vol. 105, pp. 173-178, 1986.

[19] M. J. Koren, R. B. Devereux, P. N. Casale, D. D. Savage, and J. H. Laragh, "Relation of left ventricular mass and geometry to morbidity and mortality in uncomplicated essential hypertension," Annals of Internal Medicine, vol. 114, no. 5, pp. 345-352, 1991.

[20] M. Bikkina, D. Levy, J. C. Evans et al., "Left ventricular mass and risk of stroke in an elderly cohort: the Framingham heart study," Journal of the American Medical Association, vol. 272, no. 1, pp. 33-36, 1994.

[21] Y. Shigematsu, M. Hamada, T. Ohtsuka et al., "Left ventricular geometry as an independent predictor for extracardiac target organ damage in essential hypertension," American Journal of Hypertension, vol. 11, no. 10 I, pp. 1171-1177, 1998.

[22] R. Pontremoli, M. Ravera, G. P. Bezante et al., "Left ventricular geometry and function in patients with essential hypertension and microalbuminuria," Journal of Hypertension, vol. 17, no. 7, pp. 993-1000, 1999.

[23] A. Lindgren, A. Roijer, O. Rudling et al., "Cerebral lesions on magnetic resonance imaging, heart disease, and vascular risk factors in subjects without stroke: a population-based study," Stroke, vol. 25, no. 5, pp. 929-934, 1994.

[24] W. T. Longstreth, T. A. Manolio, A. Arnold et al., "Clinical correlates of white matter findings on cranial magnetic resonance imaging of 3301 elderly people. The cardiovascular health study," Stroke, vol. 27, pp. 1274-1282, 1996.

[25] R. Schmidt, M. Hayn, F. Fazekas, P. Kapeller, and H. Esterbauer, "Magnetic resonance imaging white matter hyperintensities in clinically normal elderly individuals. Correlations with plasma concentrations of naturally occurring antioxidants," Stroke, vol. 27, no. 11, pp. 2043-2047, 1996.

[26] K. Kohara, B. Zhao, Y. Jiang et al., "Relation of left ventricular hypertrophy and geometry to asymptomatic cerebrovascular damage in essential hypertension," American Journal of Cardiology, vol. 83, no. 3, pp. 367-370, 1999.

[27] C. Sierra, A. de la Sierra, J. C. Paré, E. Gómez-Angelats, and A. Coca, "Correlation between silent cerebral white matter lesions and left ventricular mass and geometry in essential hypertension," American Journal of Hypertension, vol. 15, no. 6, pp. 507-512, 2002.

[28] E. R. Fox, H. A. Taylor Jr., E. J. Benjamin et al., "Left ventricular mass indexed to height and prevalent MRI cerebrovascular disease in an African American cohort: the atherosclerotic risk in communities study," Stroke, vol. 36, no. 3, pp. 546-550, 2005.

[29] K. Shimada, A. Kawamoto, K. Matsubayashi, and T. Ozawa, "Silent cerebrovascular disease in the elderly. Correlation with ambulatory pressure," Hypertension, vol. 16, no. 6, pp. 692699, 1990.

[30] E. J. Benjamin and D. Levy, "Why is left ventricular hypertrophy so predictive of morbidity and mortality?" American Journal of the Medical Sciences, vol. 317, no. 3, pp. 168-175, 1999.

[31] F. Fazekas, P. Kapeller, R. Schmidt, H. Offenbacher, F. Payer, and G. Fazekas, "The relation of cerebral magnetic resonance signal hyperintensities to Alzheimer's disease," Journal of the Neurological Sciences, vol. 142, no. 1-2, pp. 121-125, 1996.

[32] D. S. Knopman, T. H. Mosley, K. R. Bailey, C. R. Jack Jr., G. L. Schwartz, and S. T. Turner, "Associations of microalbuminuria with brain atrophy and white matter hyperintensities in hypertensive sibships," Journal of the Neurological Sciences, vol. 271, no. 1-2, pp. 53-60, 2008.

[33] M. Wada, H. Nagasawa, C. Iseki et al., "Cerebral small vessel disease and chronic kidney disease (CKD): results of a crosssectional study in community-based Japanese elderly," Journal of the Neurological Sciences, vol. 272, no. 1-2, pp. 36-42, 2008.

[34] D. E. Weiner, K. Bartolomei, T. Scott et al., "Albuminuria, cognitive functioning, and white matter hyperintensities in homebound elders," American Journal of Kidney Diseases, vol. 53, no. 3, pp. 438-447, 2009.

[35] C. S. Thompson and A. M. Hakim, "Living beyond our physiological means: small vessel disease of the brain is an expression of a systemic failure in arteriolar function: a unifying hypothesis," Stroke, vol. 40, no. 5, pp. e322-e330, 2009.

[36] M. G. Shlipak, L. F. Fried, C. Crump et al., "Elevations of inflammatory and procoagulant biomarkers in elderly persons with renal insufficiency," Circulation, vol. 107, no. 1, pp. 8792, 2003.

[37] A. Hassan, B. J. Hunt, M. O'Sullivan et al., "Markers of endothelial dysfunction in lacunar infarction and ischaemic leukoaraiosis," Brain, vol. 126, no. 2, pp. 424-432, 2003.

[38] C. Sierra, A. Coca, E. L. Schiffrin et al., "Vascular mechanisms in the pathogenesis of stroke," Current Hypertension Reports, vol. 13, no. 3, pp. 200-207, 2011.

[39] M. Khatri, C. B. Wright, T. L. Nickolas et al., "Chronic kidney disease is associated with white matter hyperintensity volume: the Northern Manhattan Study (NOMAS)," Stroke, vol. 38, no. 12, pp. 3121-3126, 2007.

[40] M. A. Ikram, M. W. Vernooij, A. Hofman, W. J. Niessen, A. Van Der Lugt, and M. M. B. Breteler, "Kidney function is related to cerebral small vessel disease," Stroke, vol. 39, no. 1, pp. 55-61, 2008.

[41] W. Takahashi, Y. Tsukamoto, S. Takizawa, S. Kawada, and S. Takagi, "Relationship between chronic kidney disease and white matter hyperintensities on magnetic resonance imaging," Journal of Stroke and Cerebrovascular Diseases, 2010. In press.

[42] M. L. Baker, P. J. Hand, J. J. Wang, and T. Y. Wong, "Retinal signs and stroke: revisiting the link between eye and brain," Stroke, vol. 39, no. 4, pp. 1371-1379, 2008.

[43] L. Mimoun, P. Massin, and G. Steg, "Retinal microvascularisation abnormalities and cardiovascular risk," Archives of Cardiovascular Diseases, vol. 102, no. 5, pp. 449-456, 2009.

[44] R. Klein, A. R. Sharrett, B. E. Klein et al., "Are retinal arteriolar abnormalities related to atherosclerosis? The atherosclerosis risk in communities study," Arteriosclerosis, Thrombosis, and Vascular Biology, vol. 20, no. 6, pp. 1644-1650, 2000.

[45] R. Schneider, M. Rademacher, and S. Wolf, "Lacunar infarcts and white matter attenuation: ophthalmologic and microcirculatory aspects of the pathophysiology," Stroke, vol. 24, no. 12, pp. 1874-1879, 1993.

[46] I. Goto, K. Kimoto, S. Katsuki, T. Mimatsu, and H. Ikui, "Pathological studies on the intracerebral and retinal arteries in cerebrovascular and noncerebrovascular diseases," Stroke, vol. 6, no. 3, pp. 263-269, 1975.

[47] L. D. Hubbard, R. J. Brothers, W. N. King et al., "Methods for evaluation of retinal microvascular abnormalities associated with hypertension/sclerosis in the atherosclerosis risk in communities study," Ophthalmology, vol. 106, no. 12, pp. 2269-2280, 1999.

[48] T. Y. Wong, R. Klein, A. R. Sharrett et al., "ARIC investigators. Atheroslerosis risk in communities study. Cerebral white matter lesions, retinopathy, and incident clinical stroke," Journal of the American Medical Association, vol. 288, pp. 67-74, 2002. 
[49] N. Cheung, T. Mosley, A. Islam et al., "Retinal microvascular abnormalities and subclinical magnetic resonance imaging brain infarct: a prospective study," Brain, vol. 133, no. 7, pp. 1987-1993, 2010.

[50] W. T. Longstreth, E. K. M. Larsen, R. Klein et al., "Associations between findings on cranial magnetic resonance imaging and retinal photography in the elderly: the cardiovascular health study," American Journal of Epidemiology, vol. 165, no. 1, pp. 78-84, 2007.

[51] M. K. Ikram, F. J. De Jong, E. J. Van Dijk et al., "Retinal vessel diameters and cerebral small vessel disease: the rotterdam scan study," Brain, vol. 129, no. 1, pp. 182-188, 2006.

[52] P. Prati, A. Tosetto, D. Vanuzzo et al., "Carotid intima media thickness and plaques can predict the occurrence of ischemic cerebrovascular events," Stroke, vol. 39, no. 9, pp. 2470-2476, 2008.

[53] T. A. Manolio, G. L. Burke, D. H. O’Leary et al., "Relationships of cerebral MRI findings to ultrasonographic carotid atherosclerosis in older adults: the cardiovascular health study. CHS collaborative research group," Arteriosclerosis, Thrombosis, and Vascular Biology, vol. 19, pp. 356-365, 1999.

[54] F. Pico, C. Dufouil, C. Levy et al., "Longitudinal study of carotid atherosclerosis and white matter hyperintensities: the EVA-MRI cohort," Cerebrovascular Diseases, vol. 14, no. 2, pp. 109-115, 2002.

[55] F. E. de Leeuw, J. C. de Groot, M. L. Bots et al., "Carotid atherosclerosis and cerebral white matter lesions in a population based magnetic resonance imaging study," Journal of Neurology, vol. 247, no. 4, pp. 291-296, 2000.

[56] J. R. Romero, A. Beiser, S. Seshadri et al., "Carotid artery atherosclerosis, MRI indices of brain ischemia, aging, and cognitive impairment," Stroke, vol. 40, no. 5, pp. 1590-1596, 2009.

[57] S. D. Shenkin, M. E. Bastin, T. J. MacGillivray et al., "Carotid intima-media thickness and cerebrovascular disease in community-dwelling older people without stroke," Stroke, vol. 41, no. 9, pp. 2083-2086, 2010

[58] C. Sierra, A. de la Sierra, J. Mercader, E. Gómez-Angelats, A. Urbano-Márquez, and A. Coca, "Silent cerebral white matter lesions in middle-aged essential hypertensive patients," Journal of Hypertension, vol. 20, no. 3, pp. 519-524, 2002.

[59] C. K. Kim, S. H. Lee, B. J. Kim, W. S. Ryu, and B. J. Kim, “Ageindependent association of pulse pressure with cerebral white matter lesions in asymptomatic elderly individuals," Journal of Hypertension, vol. 29, pp. 325-329, 2011.

[60] S. Laurent, S. Katsahian, C. Fassot et al., "Aortic stiffness is an independent predictor of fatal stroke in essential hypertension," Stroke, vol. 34, no. 5, pp. 1203-1206, 2003.

[61] L. H. G. Henskens, A. A. Kroon, R. J. van Oostenbrugge et al., "Increased aortic pulse wave velocity is associated with silent cerebral small-vessel disease in hypertensive patients," Hypertension, vol. 52, no. 6, pp. 1120-1126, 2008.

[62] I. Shrestha, T. Takahashi, E. Nomura et al., "Association between central systolic blood pressure, white matter lesions in cerebral MRI and carotid atherosclerosis," Hypertension Research, vol. 32, no. 10, pp. 869-874, 2009.

[63] E. J. van Dijk, N. D. Prins, S. E. Vermeer et al., "C-reactive protein and cerebral small-vessel disease: the Rotterdam scan study," Circulation, vol. 112, no. 6, pp. 900-905, 2005.

[64] M. Wada, H. Nagasawa, K. Kurita et al., "Cerebral small vessel disease and C-reactive protein: results of a cross-sectional study in community-based Japanese elderly," Journal of the Neurological Sciences, vol. 264, no. 1-2, pp. 43-49, 2008.

[65] R. Schmidt, H. Schmidt, M. Pichler et al., "C-reactive protein, carotid atherosclerosis, and cerebral small-vessel disease: results of the Austrian stroke prevention study," Stroke, vol. 37, no. 12, pp. 2910-2916, 2006.

[66] S. E. Vermeer, E. J. van Dijk, P. J. Koudstaal et al., "Homocysteine, silent brain infarcts, and white matter lesions: the Rotterdam scan study," Annals of Neurology, vol. 51, no. 3, pp. 285-289, 2002.

[67] C. B. Wright, Y. Moon, M. C. Paik et al., "Inflammatory biomarkers of vascular risk as correlates of leukoariosis," Stroke, vol. 40, no. 11, pp. 3466-3471, 2009.

[68] H. S. Markus, B. Hunt, K. Palmer, C. Enzinger, H. Schmidt, and R. Schmidt, "Markers of endothelial and hemostatic activation and progression of cerebral white matter hyperintensities: longitudinal results of the Austrian Stroke prevention study," Stroke, vol. 36, no. 7, pp. 1410-1414, 2005.

[69] F. E. de Leeuw, M. de Kleine, C. J. M. Frijns, R. Fijnheer, J. Van Gijn, and L. J. Kappelle, "Endothelial cell activation is associated with cerebral white matter lesions in patients with cerebrovascular disease," Annals of the New York Academy of Sciences, vol. 977, pp. 306-314, 2002.

[70] I. L. H. Knottnerus, J. W. P. Govers-Riemslag, K. Hamulyak et al., "Endothelial activation in lacunar stroke subtypes," Stroke, vol. 41, no. 8, pp. 1617-1622, 2010.

[71] A. S. Greenstein, R. Paranthaman, A. Burns et al., "Cerebrovascular damage in late-life depression is associated with structural and functional abnormalities of subcutaneous small arteries," Hypertension, vol. 56, no. 4, pp. 734-740, 2010.

[72] D. Rizzoni, C. De Ciuceis, E. Porteri et al., "Altered structure of small cerebral arteries in patients with essential hypertension," Journal of Hypertension, vol. 27, no. 4, pp. 838-845, 2009.

[73] D. Rizzoni, E. Porteri, G. E. M. Boari et al., "Prognostic significance of small-artery structure in hypertension," Circulation, vol. 108, no. 18, pp. 2230-2235, 2003. 


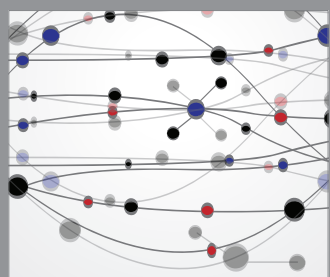

The Scientific World Journal
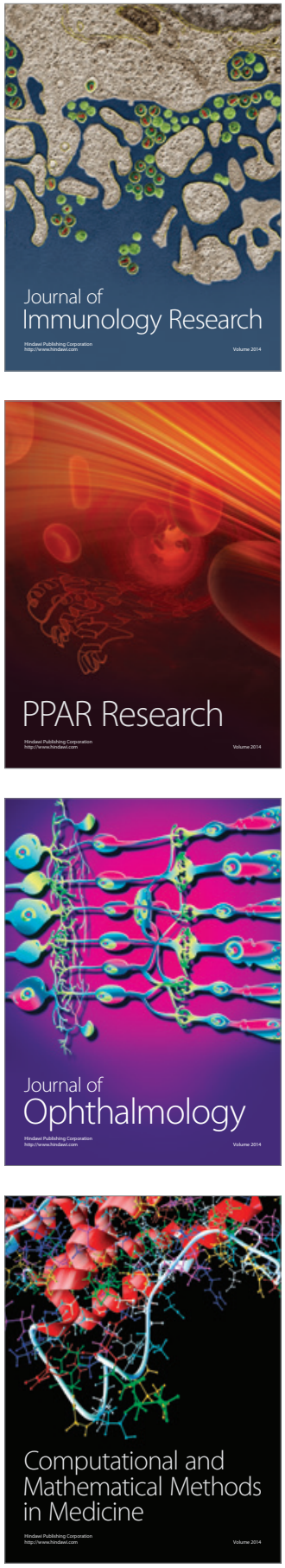

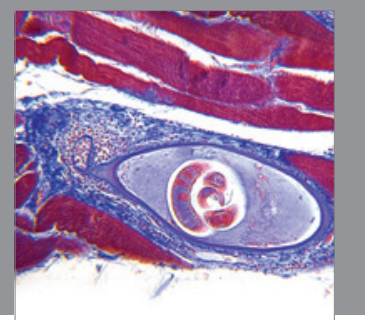

Gastroenterology

Research and Practice
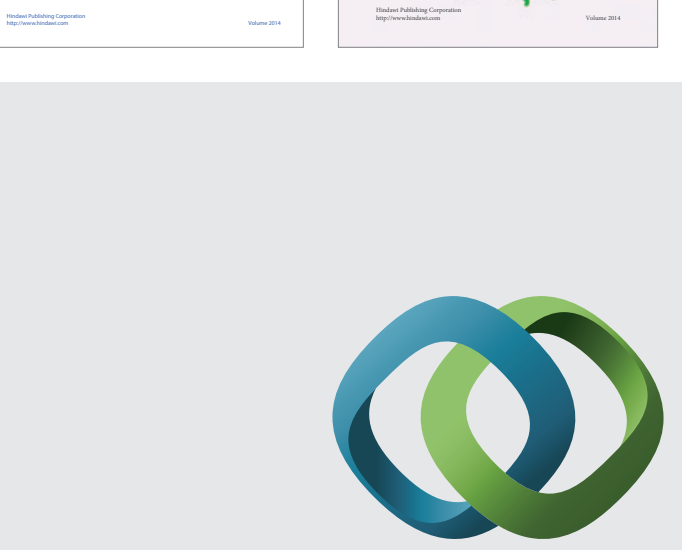

\section{Hindawi}

Submit your manuscripts at

http://www.hindawi.com
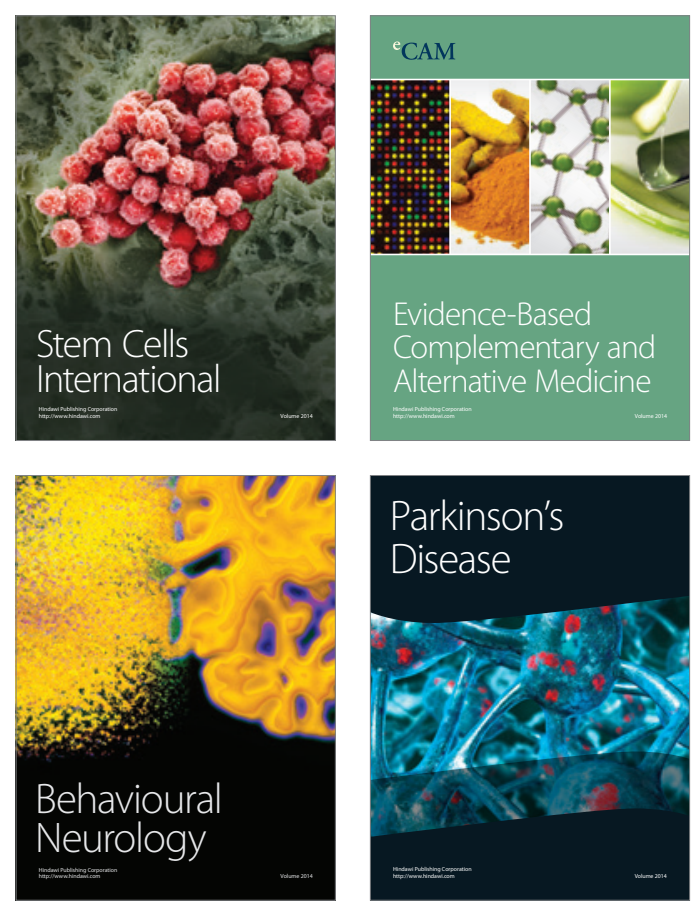

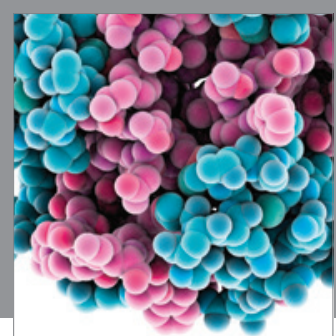

Journal of
Diabetes Research

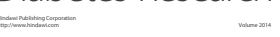

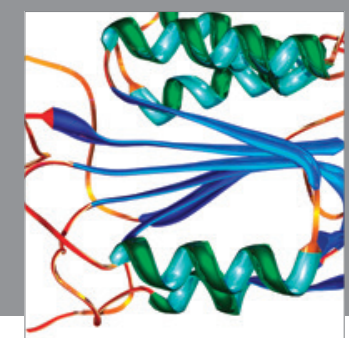

Disease Markers
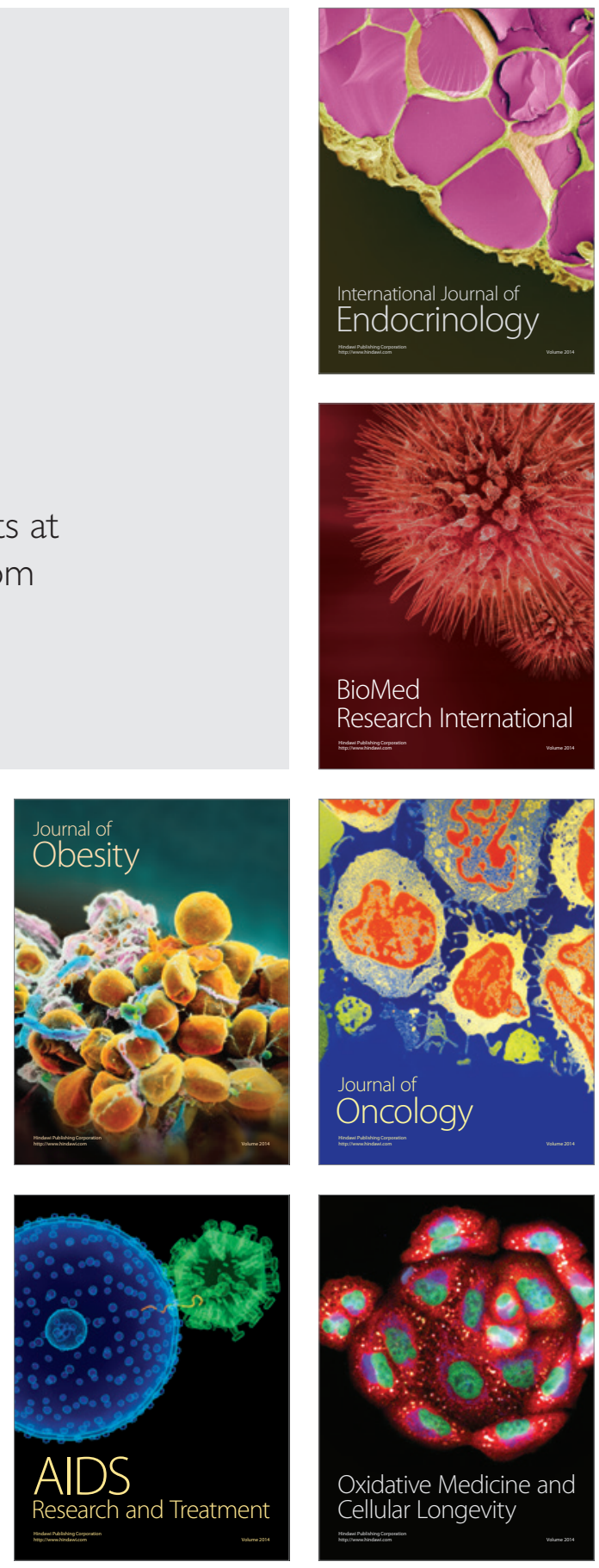